\title{
Establishment of Output Models and Constraints Analysis for Independent Wind/PV/Storage System
}

\author{
Linjing Hu, Yanjie Fu \\ College of Electric Power, Inner Mongolia University of Technology, Huhhot, China \\ Email: yanyankuaile 1989@126.com
}

Received May 2014

\begin{abstract}
In remote areas far from the grid, wind/PV/storage generating system is relatively a good choice, whatever in resource configuration, performance or prices. For the independent hybrid power system, the output models of wind turbines, photovoltaic arrays and batteries are built in this paper, and based on the objectives of the capacity configuration optimal model, constraints used in the process of capacity configuration are analyzed. These provide convenient conditions and theoretical basis for the optimal capacity configuration of independent wind/PV/storage system.
\end{abstract}

Keywords

Wind/PV/Storage System, Output Models, Constraints

\section{Introduction}

As the conventional energy sources are limited, the ever increasing human demand for energy, serious environmental pollution, global climate change, force people to seek a new, clean, safe and reliable sustainable energy systems. In recent years, many technologies of new and renewable energy have entered the stage of the commercial application, of which the greatest potential and the fastest-growing is wind and solar power generation [1]. However, wind and solar power have common weakness, such as strong randomness, poor stability, which leads to single power system's low generating efficiency and poor reliability. Wind-PV hybrid power system can be good to overcome these shortcomings. Especially in remote areas far away from the power grid, to establish an independent wind/PV/storage system is the best solution of a long-term stable and reliable power supply to local problems.

In the long term, in order to achieve the optimal economic indicators of the independent wind/PV/ storage system, reduce the difficulty of system controls, and ensure the stability of the output, reasonable capacity configuration of the system is necessary. Reliable power supply as well as minimal investment costs can be achieved with accurate and reasonable system capacity configuration [2]. The methods to optimize capacity configuration of independent wind/PV/storage system, can be divided into single-objective optimization method and multi-objectives optimization method. The former is the minimum system cost, and the latter based on sys- 
tem reliability in addition, except the cost [3]. The optimization objectives of capacity configuration is meaningful when all kinds of loads and constraints are met. Therefore, in this paper, the output models of wind turbines, photovoltaic arrays and batteries are built, and constraints used in the process of capacity configuration optimization are analyzed.

\section{Independent Wind/PV/storage Hybrid Generating System}

\subsection{Structure of the System}

Figure 1 is a block diagram of an independent wind/PV/ storage systems. The power generation system generally consists of wind turbines, photovoltaic arrays, batteries controllers, inverters, loads, etc. PV arrays and wind turbines are power generating parts. Batteries stores energy, used to balance energy supply and demand. Controllers and inverters constitute the control part of the system. Loads are the energy consumption parts [4].

The independent wind/PV/storage hybrid system takes advantage of complementarity of wind and solar, making wind, solar, battery together. Taking into account of the equipment costs, power supply system often gives priority to wind power, photovoltaic power generation as a supplement, energy storage devices playing a role in coordinating the buffer.

\subsection{Output Model of Wind Turbine}

The output of the wind on each time depends on the average wind speed at the height of the fan shaft and turbine's output characteristics [5]. Power output of wind turbines changes with wind speed, meanwhile wind has very strong randomness. Thus, statistical characteristics of wind speed can be described by its probability distribution. Two-parameter Weibull distribution is generally considered the best probability density function for wind speed statistical description.

Wind speed probability density expressed as follows [6]

$$
f(v)=\frac{k}{A}\left(\frac{v}{A}\right)^{k-1} \exp \left[-\left(\frac{v}{A}\right)^{k}\right]
$$

where

$k$ shape parameter, used to describe the dispersion of wind speed measurement;

A scale parameter, related to the average wind speed measurements;

$v$ wind speed.

When the distribution of wind speed is known, average output power of turbines can be got through the output characteristics of wind turbines. An approximate relationship between the output power of the generator and the wind speed by is described by Equation (2).

$$
P_{W}=\left\{\begin{array}{l}
P_{N} \frac{v-v_{c}}{v_{N}-v_{c}}, v_{c} \leq v \leq v_{N} \\
P_{N}, v_{N}<v \leq v_{F} \\
0, \mathrm{v}<\mathrm{v}_{c} \text { or } \mathrm{v}>v_{F}
\end{array}\right.
$$

where

$P_{W}$ output of wind turbine;

$P_{N}$ rated power of wind turbine;

$v_{c}$ cut-in speed;

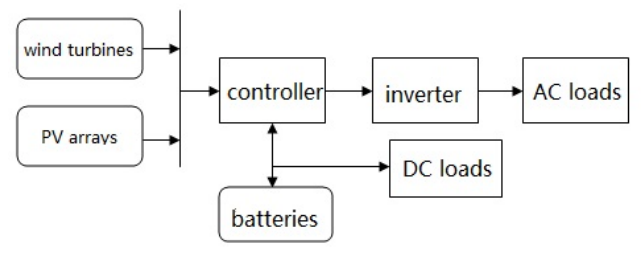

Figure 1. Block diagram of the independent wind/ PV/storage system. 
$v_{N}$ rated wind speed;

$v_{F}$ cut-out speed.

In independent wind/PV/storage hybrid generating system, the output of the wind turbines is generally used as the main power supply section, so its output forecast requires high accuracy. In this paper, output model of wind turbine using two-parameter Weibull wind speed distribution model, can make the forecast of meteorological parameters and wind power output more accurate.

\subsection{Output Model of PV Array}

In hybrid power supply system, output of PV arrays is closely related to the distribution of solar radiation energy. The solar radiant energy obtained on the specific site depends on many factors, including climate, as well as the relative positions of the Earth and the sun, obstacle interference and so on. The actual output of the PV arrays can be determined by rated power output under nominal standard conditions, light intensity, ambient temperature [7]. The steady state power output can be described as in

$$
P_{P V}=P_{S T C} G_{A C} \frac{1+\mathrm{k}\left(\mathrm{T}_{c}-\mathrm{T}_{r}\right)}{G_{S T C}}
$$

where

$P_{P V}$ output of PV cell;

$P_{S T C}$ the maximum test power under standard test conditions(solar radiation intensity $1000 \mathrm{~W} / \mathrm{m}^{2}$, ambient temperature $25^{\circ} \mathrm{C}$ );

$G_{A C}$ light intensity;

$G_{\text {STC }}$ light intensity under standard test conditions, that is $1000 \mathrm{~W} / \mathrm{m}^{2}$.

$\mathrm{k}$ power temperature coefficient;

$T_{c}$ panels working temperature;

$T_{r}$ reference temperature.

Equation (3) just calculates a single photovoltaic cell's output, which is very small. In practical engineering, PV cells usually compose high-power solar arrays after a series-parallel composition in order to meet the requirements. As a complement to wind power, the output model of PV arrays is considered to be associated just with solar radiation and ambient temperature, which is convenient for engineering applications.

\subsection{Output Model of Battery}

The independent wind/PV/storage hybrid generating system generally gives the priority to new energy. When wind-PV unit can meet the current load and has additional output, batteries charge, otherwise, batteries discharge [8]. The remaining capacity of the battery at time $t$ is related to remaining power at time $t-1$, charge and discharge in time $[t-1, t]$, and power attenuation each hour. State of charge can be expressed as in

$$
S_{o c}(t)=\mathrm{S}_{o c}(t-1)(1-\sigma) \pm \mathrm{I} \cdot \mathrm{T} \cdot \eta
$$

where

$\sigma$ self-discharge rate;

$I$ charging and discharging current;

$T$ charging and discharging time;

$\eta$ charging and discharging efficiency.

Output model of batteries describes the change of store energy state, thus the action of batteries in the operation of the system at time $t$ can be known. As an energy storage device, battery in addition to improving power quality, the larger purpose is to improve the electricity supply-demand balance [9].

\section{Capacity Configuration Optimal Model of System}

\subsection{Optimization Objectives}

This paper selects dual objectives optimization method, which can realize minimum investing and operating cost, and highest system reliability.

The objective function of the capacity configuration optimal model for independent wind/PV/storage system can be expressed as in 


$$
\left\{\begin{array}{l}
\min f_{1}\left(\mathrm{x}_{w}, x_{p}, y\right)=F_{\Sigma} \\
\max f_{2}\left(x_{w}, x_{p}, y\right)=R_{e l}
\end{array}\right.
$$

where

$$
F_{\Sigma}=\sum_{t=1}^{T}\left(C_{t}+W_{s t}\right) \cdot(1+r)^{-t}+\sum_{t=1}^{T}\left(F_{g t}+F_{k t}-B_{t}\right) \cdot(1+r)^{-(t-0.5)}
$$

$x_{w}, \quad x_{p}$ installed capacity of wind power and PV (MW) respectively;

$R_{e l}$ reliability index;

y capacity of battery;

$F_{\Sigma}$ calculated totally cost of the planning capacity of the hybrid system;

$C_{t}$ the equipment investment costs of system in year t;

$W_{\text {st }}$ grid investment costs in year $t$;

$F_{g t}, F_{k t}$ maintenance fee for fixed operation and other expenses in year $t$;

$B_{t}$ other benefits of the system in addition to electricity generation in year $t$;

$T$ the years of the planning period $(n)$;

$r$ discount rate.

\subsection{Constraints}

1) Reliability of wind turbines and photovoltaic modules selection

The correct selection of wind turbines and PV modules has respect to reliability of the system, and decides whether to take good advantage of wind and solar energy or not. Incorrect selection may cause difficulty to improve power system control, resulting in low efficiency of power generation systems and high operating costs [10].

2) System construction sites

Pre-planning construction sites of hybrid generation system are limited, so it should restrict the scale of the system according to the system construction sites, that is to say constraints on the number of devices is necessary. The number of fans erected in the field has an upper limit because of the distance of ranks of wind turbines. Moreover, considering the cover, hillsides, and unavailable areas wind towers covering, the number of PV modules installed has an upper limit, too.

3) The total capacity of power system

When configuring capacity of wind turbines, photovoltaic arrays and storage devices in a system, the total output of power system must meet the load demands. Taking into account of that the loads may increase or generating units may be faulty, the system needs to leave some spare capacity, so that generation system can be able to guarantee $\mathrm{m} \%$ power beyond that the maximum power output of the system can supply. Expression is as follows:

where

$$
P(w)+P(p v)+P(x) \geq(1+m \%) p(l)
$$

$P(w)$ annual generating capacity of wind turbines;

$P(p v)$ annual generating capacity of PV arrays;

$P(x)$ annual capacity of battery charge and discharge;

$P(l)$ annual power loads required.

4) The minimum power of generation system

Taking into account that photovoltaic has no output at night, the load power is mainly provided by wind, so that wind units must be able to provide at least average power of loads. Similarly, during the day, under weak wind or no wind condition, the power is mainly provided by the photovoltaic, so PV cells should provide at least the average power of loads. When there is no wind or sun, load power is provided by batteries, so batteries should at least ensure continuous load work.

5) Battery charge and discharge constraints

Taking into account of the life of the batteries, batteries' charging and discharging during system operating must be limited strictly. Battery charge and discharge times and depth of discharge have great effects on battery life. Depth of discharge is limited by state of charge (SOC) of the battery. State of charge must satisfy the following formula 


$$
S_{O C \min } \leq S_{O C} \leq S_{\text {OCmax }}
$$

In a scheduling cycle, the battery charge and discharge cycles cannot exceed the limit value. And the battery charge and discharge current $I_{c}$ and $I_{d}$ cannot exceed the maximum $I_{c \max }$ or $I_{d \max }$.

$$
\left\{\begin{array}{l}
0<I_{c} \leq I_{c \max } \\
0<I_{d} \leq I_{d \max }
\end{array}\right.
$$

6) Power supply performance index

The power supply goal of the independent wind/PV/storage hybrid generating system is to meet all loads in area. The loss of power supply probability (LPSP) refers to the ratio of the loss of power supply and load power consumption in normal operation in one year $(8760 \mathrm{~h})$. LPSP is used to describe the active power supply and reflect the level of power system stability and it generally used to describe the reliability of power supply [11]. Typically, loss of power supply probability should be in an appropriate range, that is

$$
f_{l p s p} \leq F_{L \max }
$$

where

$f_{\text {lpsp }}$ loss of power supply probability of the system;

$F_{L \max }$ allowable maximum LPSP of the system.

\section{Conclusion}

The independent wind/PV/storage hybrid generating system takes advantage of the complementary characteristics of wind and solar resources, which can overcome the defects of single energy generation and improve the stability of power systems. In this way, the independent wind/PV/storage hybrid generating system must develop greatly and rapidly. While reasonable capacity configuration can highlight the advantages of the system, the stability and economy in a long term can be ensured. Therefore, for the independent hybrid, the output models of wind turbines, photovoltaic arrays and batteries are built in this paper, and based on the objectives of the capacity configuration optimal model, constraints used in the process of capacity configuration are analyzed. The work provides convenient conditions to build a capacity configuration optimal model, and lays the foundation for selecting optimize algorithm.

\section{Acknowledgements}

The research funded by the following items, express my great gratitude here.

The Institutions of Higher Learning Science Research Project (NJZY13122) and the Natural Science Foundation (2013MS0707) of Inner Mongolia.

\section{References}

[1] Zhao, Y.S., Zhan, J. and Zhang, Y. (2009) The Optimal Capacity Configuration of an Independent Wind/PV Hybrid Power Supply System Based on Improved PSO Algorithm. APSCOM 8th International Conference, 1-7.

[2] Yan, F. (2009) The Optimum Match Design of Stand-Alone Hybrid Wind/PV Power System. Shandong Jianzhu University, Jinan.

[3] Lin, X., Ruan, X.B., Zhang, B.H. and Mao, C.X. (2012) An Improved Optimal Sizing Method for Wind-Solar-Battery Hybrid Power System. Proceedings of the CSEE, 5, 88-98.

[4] Chen, X.H. (2012) The Independent New Energy Hybrid Power System Optimal Capacity Configuration. Hunan University.

[5] Zhu, L., Yan, Z., Yang, X., Fu, Y. and Chen, J. (2012) Optimal Configuration of Battery Capacity in Microgrid Composed of Wind Power and Photovoltaic Generation With Energy Storage. Power System Technology, 12, $26-31$.

[6] Zhu, H., Xu, J.Y. (2008) Wind Farm Output Forecast Based on Combined GM-WEIBULL Wind Speed Distribution Models. East China Electric Power, 11, 144-146.

[7] Ma, X.Y., Wu, Y.W., Fang, H.L. and Sun, Y.Z. (2011) Optimal Sizing of Hybrid Solar-wind Distributed Generation in an Islanded Microgrid Using Improved Bacterial Foraging Algorithm. Proceedings of the CSEE, 25, 17-25.

[8] He, J., Deng, C.H., Xu, Q.S., Liu, C.L. and Pan, H. (2013) Optimal Configuration of Distributed Generation System Containing Wind/PV/Battery Power Sources Based on Equivalent Credible Capacity Theory. Power System Technol- 
ogy, 12, 3317-3324.

[9] Li, P., Liu, Y.Q. and Guo, W.Z. (2010) Study on Optimal Design Method for Stand-Alone Wind/Solar/Battery Hybrid Power System. Modern Electric Power, 12, 51-57.

[10] Zhou, K. (2012) Research on Capacity Configuration Method of Wind/PV (Diesel)/Storage Hybrid Generating System. University of Electronic Science and Technology of China.

[11] Yang, J., Zhang, J.C., Zhou, Y., Niu, H. and Liang, T.T. (2013) Research on Capacity Optimization of Hybrid Energy Storage System in Stand-Alone Wind/PV Power Generation System. Power System Protection and Control, 4, 38-43. 